\title{
The Zografos-Balakrishnan Log-Logistic Distribution: Properties and Applications
}

\author{
Manoel Wallace A. Ramos ${ }^{1, *}$, Gauss M. Cordeiro ${ }^{1}$, Pedro Rafael D. Marinho ${ }^{1}$, \\ Cícero Rafael B. Dias ${ }^{1}$ and G. G. Hamedani ${ }^{2}$ \\ ${ }^{1}$ Departamento de Estatística, Universidade Federal de Pernambuco, \\ Recife, PE, Brazil \\ ${ }^{2}$ Department of Mathematics, Statistics and Computer Science, \\ Marquette University, Milwaukee, USA \\ Received 12 September 2012 \\ Accepted 31 March 2013
}

\begin{abstract}
The log-logistic distribution (also known as the Fisk distribution in economics) is widely used in survival analysis when the failure rate function presents a unimodal shape. In this paper, we introduce the ZografosBalakrishnan log-logistic distribution, which contains the log-logistic distribution as a special model and has the four common shapes of the hazard hate function. We present some properties of the new distribution and estimate the model parameters by maximum likelihood. An application to a real data set shows that the new distribution can provide a better fit than other classical lifetime models such as the exponentiated Weibull distribution.
\end{abstract}

Keywords: Estimation; Generating function; Log-logistic distribution; Mean deviation; Moment; ZografosBalakrishnan log-logistic distribution.

AMS 2000 Subject Classification: 62E99; 60E05

\section{Introduction}

Zografos and Balakrishnan [26] and Ristic and Balakrishnan [24] proposed different families of univariate distributions generated by gamma random variables. For any baseline cumulative distribution function (cdf) $G(x)$, and $x \in \mathbb{R}$, Zografos and Balakrishnan [26] defined the ZografosBalakrishnan-G (ZB-G) distribution with probability density function (pdf) $f(x)$ and $\operatorname{cdf} F(x)$ (for $a>0$ ) given by

$$
f(x)=\frac{1}{\Gamma(a)}\{-\log [1-G(x)]\}^{a-1} g(x)
$$

\footnotetext{
*Correspondence: Manoel Wallace A. Ramos, Departamento de Estatística, Universidade Federal de Pernambuco, Cidade Universitária, 50740-540 Recife, PE, Brazil. E-mail: wallace.ifpb@gmail.com
} 
and

$$
F(x)=\frac{\gamma(a,-\log [1-G(x)])}{\Gamma(a)}=\frac{1}{\Gamma(a)} \int_{0}^{-\log [1-G(x)]} t^{a-1} \mathrm{e}^{-t} d t
$$

respectively, where $g(x)=d G(x) / d x, \Gamma(a)=\int_{0}^{\infty} t^{a-1} \mathrm{e}^{-t} d t$ denotes the gamma function, and $\gamma(a, z)=\int_{0}^{z} t^{a-1} \mathrm{e}^{-t} d t$ denotes the incomplete gamma function. The corresponding hazard rate function (hrf) is

$$
h(x)=\frac{\{-\log [1-G(x)]\}^{a-1} g(x)}{\Gamma(a,-\log [1-G(x)])},
$$

where $\Gamma(a, z)=\int_{z}^{\infty} t^{a-1} \mathrm{e}^{-t} d t$ denotes the complementary incomplete gamma function. On the other hand, Ristic and Balakrishnan [24] defined the Ristic-Balakrishnan-G (RB-G) distribution for $x \in$ $\mathbb{R}, a>0$ with pdf and survival function given by

$$
f(x)=\frac{1}{\Gamma(a)}\{-\log G(x)\}^{a-1} g(x) \text { and } \bar{F}(x)=\frac{1}{\Gamma(a)} \int_{0}^{-\log G(x)} t^{a-1} \mathrm{e}^{-t} d t,
$$

respectively. In this paper, we shall work only with the family (1.1). The ZB-G distribution has the same parameters of the $\mathrm{G}$ distribution plus an additional shape parameter $a>0$. If $X$ is a random variable with pdf (1.1), we write $X \sim \mathrm{ZB}-\mathrm{G}(a)$. Each new ZB-G distribution can be obtained from a specified $\mathrm{G}$ distribution. For $a=1$, the $\mathrm{G}$ distribution is a basic exemplar of the ZB-G distribution with a continuous crossover towards cases with different shapes (for example, a particular combination of skewness and kurtosis). Zografos and Balakrishnan [26] motivated the ZB-G model as follows. Let $X_{(1)}, X_{(2)}, \ldots, X_{(n)}$ be lower record values from a sequence of i.i.d. random variables from a population with pdf $g(x)$. Then, the pdf of the $n$th lower record value is given by (1.1). A logarithmic transformation of the parent distribution $\mathrm{G}$ transforms the random variable $X$ with density (1.1) to a gamma distribution. That is, if $X$ has the density (1.1), then the random variable $Z=-\log [1-G(X)]$ has a gamma distribution $G(a, 1)$ with density $\pi(z ; a)=z^{a-1} \mathrm{e}^{-z} / \Gamma(a), z>0$. The opposite is also true, if $Z$ has a gamma $G(a, 1)$ distribution, then the random variable $X=G^{-1}\left(1-\mathrm{e}^{-z}\right)$ has a ZB-G distribution (1.1). Nadarajah et al. [23] derived some mathematical properties of (1.1) and (1.2) in the most simple, explicit and general forms for the ZB-G distributions. They obtained general expressions for shape and asymptotic properties of (1.1), (1.2) and (1.3), quantile function, ordinary and incomplete moments, moment generating function (mgf), mean deviations, Bonferroni and Lorenz curves, asymptotic distribution of the extreme values, Shannon entropy, Rényi entropy, reliability and some properties of the order statistics.

In this paper, we use the generator suggested by Zografos and Balakrishnan [26] to define a new model, so-called the Zografos-Balakrishnan log-logistic (ZBLL) distribution, which generalizes the log-logistic (LL) distribution. In addition, we investigate some structural properties of 
the new model, discuss maximum likelihood estimation of its parameters and derive the observed information matrix. The proposed model is much more flexible than the LL distribution and can be used effectively for modeling positive real data. The rest of the paper is organized as follows. In Section 2, we define the new distribution and derive useful expansions. In Section 3, explicit expressions for the ordinary and incomplete moments, generating and quantile functions, mean deviations, Rényi entropy and reliability are derived. Characterizations of the ZBLL model are described in Section 4. The order statistics are investigated in Section 5. Estimation of the model parameters by the method of maximum likelihood is presented in Section 6. An application to a real data set is illustrated in Section 7. Finally, some conclusions and future work are addressed in Section 8 .

\section{The ZBLL model and useful expansions}

The pdf and cdf of the LL distribution are (for $x, \alpha, \beta>0$ )

$$
g(x ; \alpha, \beta)=\frac{\beta}{\alpha^{\beta}} x^{\beta-1}\left[1+\left(\frac{x}{\alpha}\right)^{\beta}\right]^{-2}, \quad G(x ; \alpha, \beta)=1-\left[1+\left(\frac{x}{\alpha}\right)^{\beta}\right]^{-1},
$$

respectively, where $\alpha>0$ is a scale parameter and $\beta>0$ is a shape parameter. This distribution is widely used in practice and it is an alternative to the log-normal distribution since it presents a failure rate function that increases, reaches a peak after some finite period and then declines gradually. Some properties and applications of the LL distribution were addressed by Kleiber and Kotz [15] and Ashkar and Mahdi [1]. Its moments are given by (Tadikamalla, [25])

$$
E\left(T^{s}\right)=\alpha^{s} B\left(1-\frac{s}{\beta}, 1+\frac{s}{\beta}\right)=\frac{s \pi \alpha \beta^{-1}}{\sin \left(s \pi \beta^{-1}\right)}, s<\beta
$$

where $B(a, b)=\int_{0}^{1} w^{a-1}(1-w)^{b-1} d w$ is the beta function. Inserting equations (2.1) into (1.1) and (1.2) gives the pdf and cdf of the ZBLL distribution (for $x>0$ )

$$
f(x)=\frac{\beta}{\alpha^{\beta} \Gamma(a)} x^{\beta-1}\left[1+\left(\frac{x}{\alpha}\right)^{\beta}\right]^{-2}\left\{\log \left[1+\left(\frac{x}{\alpha}\right)^{\beta}\right]\right\}^{a-1}
$$

and

$$
F(x)=\frac{\gamma\left(a, \log \left[1+\left(\frac{x}{\alpha}\right)^{\beta}\right]\right)}{\Gamma(a)}=\frac{1}{\Gamma(a)} \int_{0}^{\log \left[1+\left(\frac{x}{\alpha}\right)^{\beta}\right]} t^{a-1} \mathrm{e}^{-t} d t,
$$

respectively. The LL distribution is a basic exemplar for $a=1$. Plots of the ZBLL density and hazard rate functions for selected parameter values are displayed in Figures 1 and 2, respectively. We note that the ZBLL hrf can be increasing, decreasing, bathtub and unimodal, whereas the LL hrf is only unimodal. A random variable $X$ having density function (2.2) is denoted by $X \sim \operatorname{ZBLL}(a, \alpha, \beta)$. 
(a)

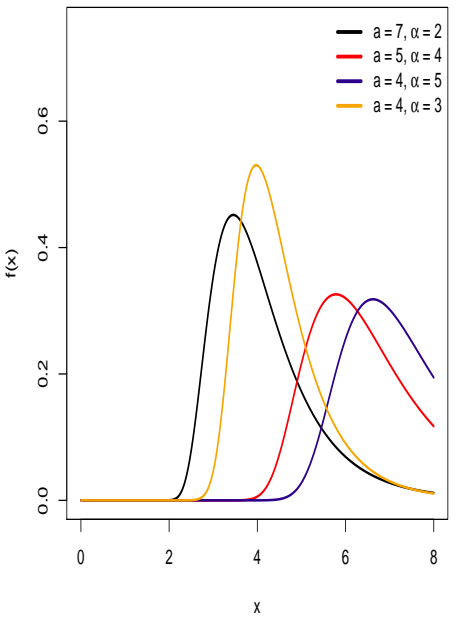

(b)

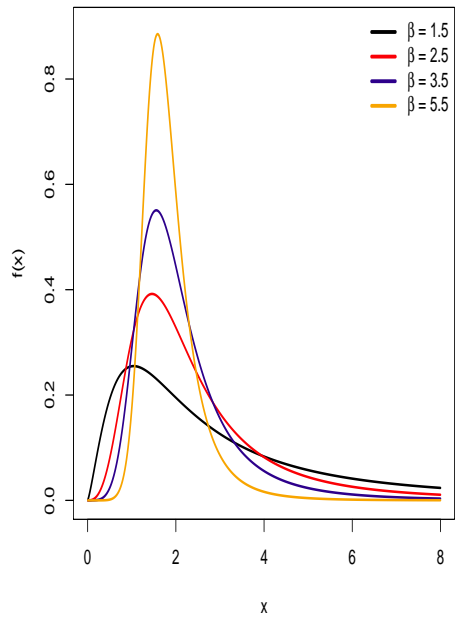

(c)

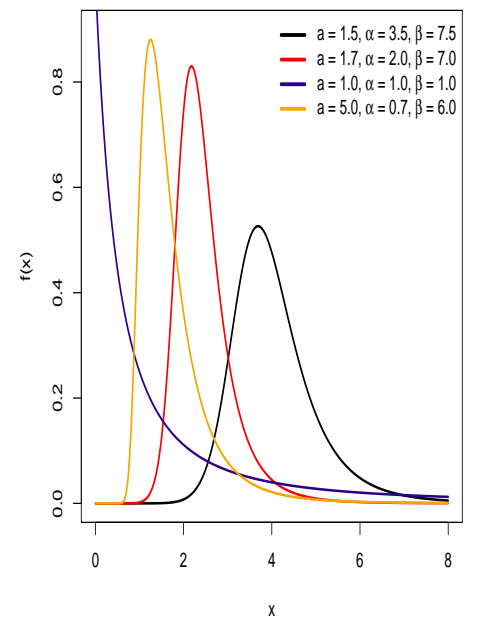

Fig. 1. The ZBLL density function for some parameter values: (a) $\beta=10$; (b) $a=1.5$ and $\alpha=1.5$.

(a)

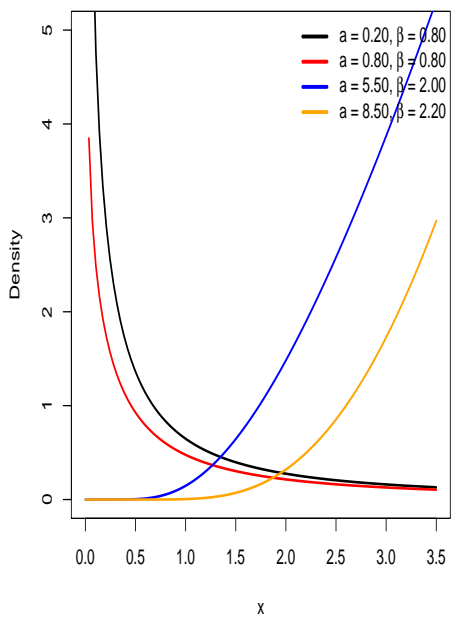

(b)

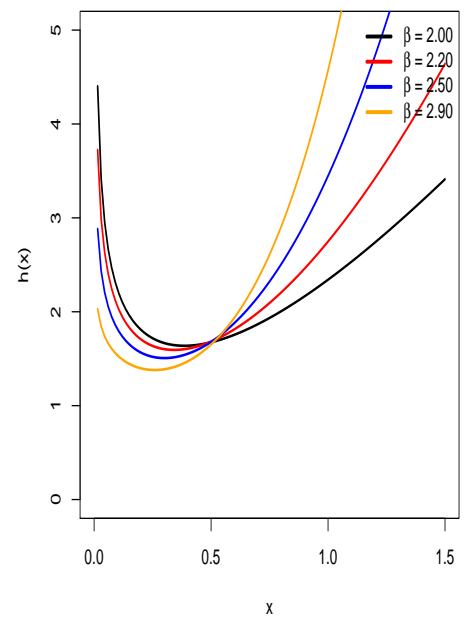

(c)

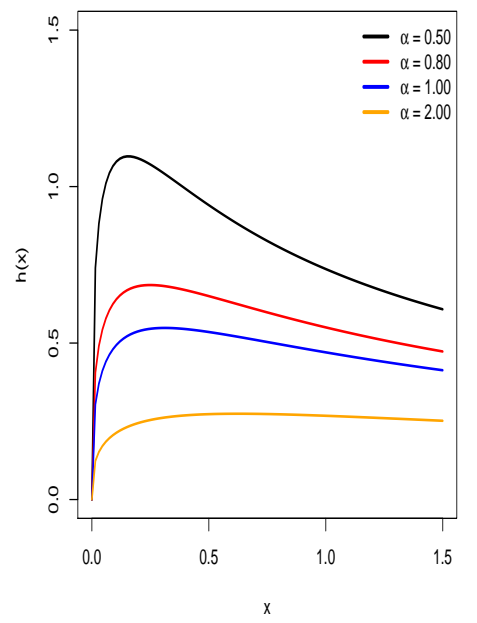

Fig. 2. The ZBLL hazard rate function for some parameter values: (a) $\alpha=0.5$; (b) $a=0.30$ and $\alpha=0.8$; (c) $a=1.2$ and $\beta=1.1$.

The explicit expressions derived throughout the paper can be easily handled in most computation software platforms such as Maple, Mathematica and Matlab. These platforms have currently the ability to deal with analytic formula of formidable size and complexity. Established explicit expressions to calculate statistical measures can be more efficient than computing them directly by 
numerical integration. The infinity limit in the sums can be substituted by a large positive integer such as 20 or 30 for most practical purpose.

Some useful expansions for (1.1) and (1.2) can be derived using the concept of exponentiated distributions. For an arbitrary baseline $\operatorname{cdf} G(x)$, a random variable is said to have the exponentiated$\mathrm{G}$ ("exp-G") distribution with parameter $a>0$, say $X \sim \exp -\mathrm{G}(a)$, if its pdf and cdf are

$$
h_{a}(x)=a G^{a-1}(x) g(x) \text { and } H_{a}(x)=G^{a}(x),
$$

respectively. The properties of exponentiated distributions have been studied by many authors in recent years, see Mudholkar and Srivastava [19] for exponentiated Weibull, Gupta et al. [9] for exponentiated Pareto, Gupta and Kundu [10] for exponentiated exponential, Nadarajah [21] for exponentiated Gumbel, among several others. Nadarajah et al. [23] demonstrated that (1.1) can be expressed as

$$
f(x)=\sum_{k=0}^{\infty} b_{k} h_{a+k}(x)
$$

where

$$
b_{k}=\frac{\left(\begin{array}{c}
k+1-a \\
k
\end{array}\right)}{(a+k) \Gamma(a-1)} \sum_{j=0}^{k} \frac{(-1)^{j+k}\left(\begin{array}{l}
k \\
j
\end{array}\right) p_{j, k}}{(a-1-j)},
$$

where the constants $p_{j, k}$ can be calculated recursively by

$$
p_{j, k}=k^{-1} \sum_{m=1}^{k} \frac{(-1)^{m}[m(j+1)-k]}{(m+1)} c_{m} p_{j, k-m}
$$

for $k=1,2, \ldots$ and $p_{j, 0}=1$. Here, $h_{a+k}(x)$ denotes the pdf of the exp-G $(a+k)$ distribution. The cdf corresponding to (2.5) is $F(x)=\sum_{k=0}^{\infty} b_{k} H_{a+k}(x)$, where $H_{a+k}(x)$ denotes the cdf of the exp$G(a+k)$ distribution. So, several mathematical properties of the ZB-G distribution can be obtained by knowing those of the exp-G distribution, see, for example, Mudholkar et al. [20], Gupta and Kundu [11] and Nadarajah and Kotz [22], among others.

\section{Properties of the ZBLL distribution}

\subsection{Moments}

Hereafter, let $Y_{a+k} \sim \operatorname{ELL}(a+k)$ denotes the exponentiated log-logistic (ELL) random variable with power parameter $a+k$. The sth moment of $X$ can be obtained from (2.5) as

$$
E\left(X^{s}\right)=\sum_{k=0}^{\infty} b_{k} E\left(Y_{a+k}^{s}\right)
$$


M. W. A. Ramos et al.

where the sth moment of $Y_{a+k}$ is given by (for $s<\beta$ ) (Lemonte, 2012) $E\left(Y_{a+k}^{s}\right)=(a+k) \alpha^{s} B(1-$ $s / \beta, a+k+s / \beta)$. Then, the $s$ th moment of $X$ can expressed as

$$
E\left(X^{s}\right)=\alpha^{s} \sum_{k=0}^{\infty}(a+k) b_{k} B\left(1-\frac{s}{\beta}, a+k+\frac{s}{\beta}\right) .
$$

The skewness and kurtosis measures can be calculated from the ordinary moments using wellknown relationships. Plots of the skewness and kurtosis for some choices of $\alpha$ and $\beta$ as functions of $a$, and for some choices of $a$ and $\alpha$ as functions of $\beta$ are displayed in Figure 3. These plots indicate that there is a great flexibility of the skewness and kurtosis curves of the new distribution.

\subsection{Quantile and generating functions}

The quantile function for the ZB-G distribution can be expressed for $(0<u<1)$ (Nadarajah et al., [23]) as $F^{-1}(u)=G^{-1}\left\{1-\exp \left[-Q^{-1}(a, 1-u)\right]\right\}$, where $Q^{-1}(a, u)$ is the inverse function of $Q(a, x)=1-\gamma(a, x) / \Gamma(a)$, which is available in several statistical packages, and $G^{-1}(u)$ is the quantile function of the baseline cdf $G(x)$. Using the LL quantile function, the ZBLL quantile function reduces to

$$
F^{-1}(u)=\alpha\left\{\exp \left[Q^{-1}(a, 1-u)\right]-1\right\}^{1 / \beta}, 0<u<1 .
$$

Here, we derive a formula for the moment generating function (mgf) $M(t)=E\left(\mathrm{e}^{t X}\right)$ of $X$. From equation (2.5), we obtain

$$
M(t)=\sum_{k=0}^{\infty}(a+k) b_{k} \rho(t, a+k-1),
$$

where the function

$$
\rho(t, a)=\int_{-\infty}^{\infty} \exp (t x) G(x)^{a} g(x) d x=\int_{0}^{1} \exp \left\{t Q_{G}(u)\right\} u^{a} d u
$$

can be determined from the baseline quantile function $Q_{G}(x)=G^{-1}(x)$. Then, for $\beta>1, M(t)$ can be expressed as

$$
M(t)=\alpha \mathrm{e}^{t} \sum_{k=0}^{\infty}(a+k) b_{k} B\left(k+a+\frac{1}{\beta}, 1-\frac{1}{\beta}\right) .
$$

\subsection{Incomplete Moments}

These types of moments play an important role for measuring inequality, for example, income quantiles and Lorenz and Bonferroni curves, which depend upon the incomplete moments of the distribution. The $r$ th incomplete moment of $X$ is defined as $m_{r}(z)=\int_{-\infty}^{z} x^{r} f(x) d x$. By inserting 

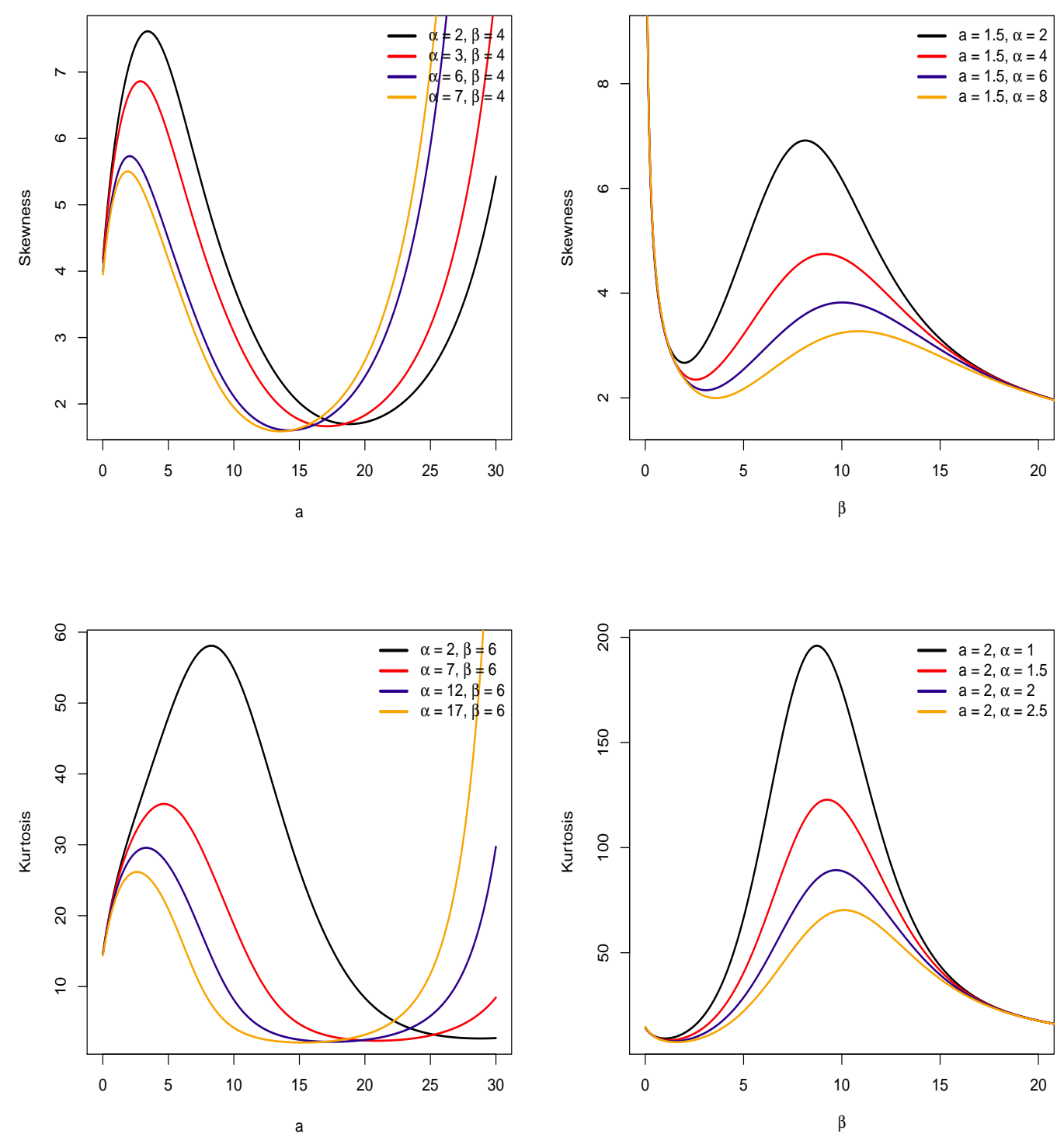

Fig. 3. Plots of the ZBLL skewness and kurtosis as functions of $a$ for selected values of $\alpha$ and $\beta$ and as functions of $\beta$ for selected values of $a$ and $\alpha$.

(2.5) in this integral, we obtain

$$
m_{r}(z)=\sum_{k=0}^{\infty} \frac{\beta(a+k) b_{k} z^{r+\beta(a+k)}}{[r+(a+k) \beta]}{ }_{2} F_{1}\left[1+a+k, a+k+\frac{r}{\beta}, 1+a+k+\frac{r}{\beta},-\left(\frac{z}{\alpha}\right)^{\beta}\right]
$$

where ${ }_{2} F_{1}$ is the hipergeometric function defined by

$$
{ }_{2} F_{1}(a, b ; c ; x)=\sum_{k=0}^{\infty} \frac{(a)_{k}(b)_{k}}{(c)_{k}} \frac{x^{k}}{k !}
$$


M. W. A. Ramos et al.

and $(a)_{k}=a(a+1) \ldots(a+k-1)$ denotes the ascending factorial.

The main application of the first incomplete moment is to derive the mean deviations about the mean and about the median of $X$ from the relations $\delta_{1}(X)=E\left(\left|X-\mu_{1}^{\prime}\right|\right)=2 \mu_{1}^{\prime} F\left(\mu_{1}^{\prime}\right)-2 m_{1}\left(\mu_{1}^{\prime}\right)$ and $\delta_{2}(X)=E(|X-M|)=\mu_{1}^{\prime}-2 m_{1}(M)$, respectively, where $\mu_{1}^{\prime}=E(X)$ comes from (3.1) with $s=1$, the median $M$ comes from (3.2) as $M=\alpha\left\{\exp \left[Q^{-1}(a, 1 / 2)\right]-1\right\}^{1 / \beta}, F\left(\mu_{1}^{\prime}\right)$ is easily obtained from (2.3) and $m_{1}(\cdot)$ is determined from (3.4) with $r=1$.

The mean deviations are useful to obtain the Bonferroni (B) and Lorenz (L) curves which have applications in several areas. For a positive random variable $X$, they are defined by $B(\pi)=$ $m_{1}(q) /\left(\pi \mu_{1}^{\prime}\right)$ and $L(\pi)=m_{1}(q) / \mu_{1}^{\prime}$, where $q=F^{-1}(\pi)=Q(\pi)$ is computed from (3.2). The measures $B(\pi)$ and $L(\pi)$ are determined from equation (3.4) using $m_{1}(Q(\pi))$.

\subsection{Rényi Entropy}

The entropy of a random variable $\mathrm{X}$ with density function $f(x)$ is a measure of variation of the uncertainty. The Rényi entropy is defined as $I_{R}(\gamma)=(1-\gamma)^{-1} \log \left(\int_{0}^{\infty} f^{\gamma}(x) d x\right)$ for $\gamma>0$ and $\gamma \neq 1$. For $a \neq 1$, the Rényi entropy of the ZB-G distribution is given by (Nadarajah et al., [23])

$$
\begin{aligned}
I_{R}(\gamma) & =-\frac{\gamma \log \Gamma(a)}{(1-\gamma)}+\frac{1}{(1-\gamma)} \log \left\{(a-1) \gamma \sum_{k=0}^{\infty}\left(\begin{array}{c}
k-\gamma a+\gamma \\
k
\end{array}\right)\right. \\
& \left.\times \sum_{j=0}^{k} \frac{(-1)^{j+k} p_{j, k}}{[\gamma(a-1)-j]}\left(\begin{array}{c}
k \\
j
\end{array}\right) M_{k}\right\},
\end{aligned}
$$

where $M_{k}=\int_{0}^{\infty} G(x)^{[\gamma(a-1)+k]} g^{\gamma}(x) d x$, and the constants $p_{j, k}$ are defined in Section 2. After some algebra, we obtain (for $a \neq 1$ )

$$
I_{R}(\gamma)=-\frac{\gamma \log \Gamma(a)}{(1-\gamma)}+\frac{1}{(1-\gamma)} \log \left\{\sum_{k=0}^{\infty} s_{k} B\left(\frac{\gamma(\beta+1)-1}{\beta}, \frac{\gamma(\beta a-1)+\beta k+1}{\beta}\right)\right\},
$$

where

$$
s_{k}=(a-1) \gamma \beta^{\gamma-1} \alpha^{\gamma(2 a \beta+2 \beta-\gamma)+1}\left(\begin{array}{c}
k-\gamma a+\gamma \\
k
\end{array}\right) \sum_{j=0}^{k} \frac{(-1)^{j+k}\left(\begin{array}{l}
k \\
j
\end{array}\right) p_{j, k}}{[\gamma(a-1)-j]} .
$$

The proof of (3.6) is given in Appendix A.

\subsection{Reliability}

Here, we derive the reliability, $R=\operatorname{Pr}\left(X_{2}<X_{1}\right)$, when $X_{1} \sim \operatorname{ZBLL}\left(a_{1}, \alpha, \beta\right)$ and $X_{2} \sim$ $\operatorname{ZBLL}\left(a_{2}, \alpha, \beta\right)$ are independent random variables. Probabilities of this form have many applications especially in engineering concepts. Let $f_{i}$ denote the pdf of $X_{i}$ and $F_{i}$ denote the cdf of $X_{i}$. 
Based on the representation (2.5) and the corresponding cdf, we can write

$$
R=\sum_{j, k=0}^{\infty} c_{j k} \int_{0}^{\infty} H_{a_{2}+j}(x) h_{a_{1}+k}(x) d x=\sum_{j, k=0}^{\infty} c_{j k} R_{j k}
$$

where

$$
\begin{gathered}
c_{j k}=\frac{\left(\begin{array}{c}
k+1-a_{1} \\
k
\end{array}\right)}{\left(a_{1}+k\right) \Gamma\left(a_{1}-1\right)} \frac{\left(\begin{array}{c}
j+1-a_{2} \\
j
\end{array}\right)}{\left(a_{2}+j\right) \Gamma\left(a_{2}-1\right)} I_{k}\left(a_{1}\right) I_{j}\left(a_{2}\right), \\
I_{k}\left(a_{1}\right)=\sum_{i=0}^{k} \frac{(-1)^{i+k} p_{i, k}}{\left(a_{1}-1-i\right)}\left(\begin{array}{c}
k \\
i
\end{array}\right),
\end{gathered}
$$

and $R_{j k}=\operatorname{Pr}\left(Y_{j}<Y_{k}\right)$ is the reliability for the independent random variables $Y_{j} \sim \operatorname{ELL}\left(a_{2}+j\right)$ and $Y_{k} \sim \operatorname{ELL}\left(a_{1}+k\right)$. Hence, the reliability of the ZBLL distribution is a linear combination of the ELL reliabilities. For the special case $a_{1}=a_{2}$, equation (3.7) reduces to $R=1 / 2$. We can obtain after some algebra

$$
R=\sum_{j, k=0}^{\infty} \frac{\left(a_{1}+k\right) c_{j k} \alpha^{-\beta\left(a_{1}+a_{2}+j+k+1\right)}}{\left(a_{1}+a_{2}+j+k\right)} .
$$

The proof of (3.8) is given in Appendix B.

\section{Characterization results}

The problem of characterizing a distribution is an important problem which has recently attracted the attention of many researchers. Thus, various characterizations have been established in many different directions. In practice, an investigator will be vitally interested to know if their model fits the requirements of their proposed distribution. To this end, the investigator relies on the characterizations of the distribution which provide conditions under which the underlying distribution is indeed the proposed distribution

Here, we present characterizations of the ZBLL distribution with pdf (2.2) in terms of: $(i)$ a simple relationship between two truncated moments; (ii) truncated moments of certain functions of the $n^{\text {th }}$ order statistic; (iii) truncated moments of certain functions of the $1^{\text {st }}$ order statistic. We cite here the works of Galambos and Kotz [4], Kotz and Shanbhag [16], Glänzel et al. [7], Glänzel [5], Glänzel and Win [8], Glänzel and Hamedani [6] and Hamedani [12-14] in these directions. Due to the format of the ZBLL cdf, we believe characterizations in other directions may not be possible or if possible will be quite complicated.

\subsection{Based on the ratio of two truncated moments}

Our first set of characterizations will employ an interesting result due to Glänzel [5] (Theorem 4.1 below). 
Theorem 4.1. Let $(\Omega, \mathfrak{F}, \mathbf{P})$ be a given probability space and let $H=[a, b]$ be an interval for some $a<b(a=-\infty, b=\infty$ might as well be allowed $)$. Let $X: \Omega \rightarrow H$ be a continuous random variable with $c d f F$ and let $g$ and $h$ be two real functions defined on $H$ such that:

$$
E[g(X) \mid X \geq x]=E[h(X) \mid X \geq x] \eta(x), \quad x \in H,
$$

is defined with some real function $\eta$. Assume that $g, h \in C^{1}(H), \eta \in C^{2}(H)$ and $F$ is twice continuously differentiable and strictly monotone function on the set $H$. Finally, assume that the equation $h \eta=g$ has no real solution in the interior of $H$. Then, $F$ is uniquely determined by the functions $g$, $h$ and $\eta$, namely

$$
F(x)=C \int_{a}^{x}\left|\frac{\eta^{\prime}(u)}{\eta(u) h(u)-g(u)}\right| \exp [-s(u)] d u,
$$

where the function $s$ is a solution of the differential equation $s^{\prime}=\frac{\eta^{\prime} h}{\eta h-g}$ and $C$ is a constant chosen to make $\int_{H} d F=1$.

We like to mention that this kind of characterization based on the ratio of truncated moments is stable in the sense of weak convergence, in particular, let us assume that there is a sequence $\left\{X_{n}\right\}$ of random variables with distribution functions $\left\{F_{n}\right\}$ such that the functions $g_{n}, h_{n}$ and $\eta_{n}(n \in \mathbb{N})$ satisfy the conditions of Theorem 1 and let $g_{n} \rightarrow g, h_{n} \rightarrow h$ for some continuously differentiable real functions $g$ and $h$. Let $X$ be a random variable with distribution $F$. Under the condition that $g_{n}(X)$ and $h_{n}(X)$ are uniformly integrable and that the family is relatively compact, the sequence $X_{n}$ converges to $X$ in distribution if and only if $\eta_{n}$ converges to $\eta$, where

$$
\eta(x)=\frac{E[g(X) \mid X \geq x]}{E[h(X) \mid X \geq x]}
$$

This stability theorem makes sure that the convergence of distribution functions is reflected by corresponding convergence of the functions $g, h$ and $\eta$, respectively. It guarantees, for instance, the "convergence" of characterization of the Wald distribution to that of the Lévy-Smirnov distribution if $\alpha \rightarrow \infty$.

A further consequence of the stability property of Theorem 4.1 is the application of this theorem to special tasks in statistical practice such as the estimation of the parameters of discrete distributions. For such purpose, the functions $g, h$ and, specially, $\eta$ should be as simple as possible. Since the function triplet is not uniquely determined it is often possible to choose $\eta$ as a linear function. Therefore, it is worth analyzing some special cases which helps to find new characterizations reflecting the relationship between individual continuous univariate distributions and appropriate in other areas of statistics. 
Remark 4.1. (a) In Theorem 4.1, the interval $H$ need not be closed. (b) The goal is to have the function $\eta$ as simple as possible. For a more detailed discussion on the choice of $\eta$, we refer the reader to Glänzel and Hamedani [6] and Hamedani [12-14].

Proposition 4.1. Let $X: \Omega \rightarrow \mathbb{R}^{+}$be a continuous random variable and let $h(x)=2$ $\left\{\log \left[1+\left(\frac{x}{\alpha}\right)^{\beta}\right]\right\}^{1-a}\left[1+\left(\frac{x}{\alpha}\right)^{\beta}\right]^{-1}$ and $g(x)=\left\{\log \left[1+\left(\frac{x}{\alpha}\right)^{\beta}\right]\right\}^{1-a}$ for $x>0$. The pdf of $X$ is (2.2) if and only if the function $\eta$ defined in Theorem 4.1 has the form

$$
\eta(x)=\left[1+\left(\frac{x}{\alpha}\right)^{\beta}\right], x>0 .
$$

Proof. Let $X$ have pdf (2.2). Then,

$$
[1-F(x)] \mathbf{E}[h(X) \mid X \geq x]=\frac{1}{\Gamma(a)}\left[1+\left(\frac{x}{\alpha}\right)^{\beta}\right]^{-2}, \quad x>0
$$

and

$$
[1-F(x)] \mathbf{E}[g(X) \mid X \geq x]=\frac{1}{\Gamma(a)}\left[1+\left(\frac{x}{\alpha}\right)^{\beta}\right]^{-1}, \quad x>0
$$

where $F$ is the cdf corresponding to the pdf $f$. Finally,

$$
\eta(x) h(x)-g(x)=\left\{\log \left[1+\left(\frac{x}{\alpha}\right)^{\beta}\right]\right\}^{1-a}>0 \text { for } x>0
$$

Conversely, if $\eta$ is given as above, we have

$$
s^{\prime}(x)=\frac{\eta^{\prime}(x) h(x)}{\eta(x) h(x)-g(x)}=\frac{2 \beta}{\alpha^{\beta}} x^{\beta-1}\left[1+\left(\frac{x}{\alpha}\right)^{\beta}\right]^{-1}, x>0,
$$

and hence $s(x)=\log \left\{\left[1+\left(\frac{x}{\alpha}\right)^{\beta}\right]\right\}^{2}, x \in \mathbb{R}^{+}$. Now, in view of Theorem 4.1 (with $C$ chosen appropriately), $X$ has pdf (2.2).

Remark 4.2. Clearly, there are other triplets $(h, g, \eta)$ satisfying the conditions of Proposition 4.1.

Corollary 4.1. Let $X: \Omega \rightarrow \mathbb{R}^{+}$be a continuous random variable and let $h(x)=2$ $\left\{\log \left[1+\left(\frac{x}{\alpha}\right)^{\beta}\right]\right\}^{1-a}\left[1+\left(\frac{x}{\alpha}\right)^{\beta}\right]^{-1}$ for $x \in \mathbb{R}^{+}$. The pdf of $X$ is (2.2) if and only if there exist functions $g$ and $\eta$ defined in Theorem 4.1 satisfying the differential equation 


$$
\frac{\eta^{\prime}(x) 2\left\{\log \left[1+\left(\frac{x}{\alpha}\right)^{\beta}\right]\right\}^{1-a}\left[1+\left(\frac{x}{\alpha}\right)^{\beta}\right]^{-1}}{\eta(x) 2\left\{\log \left[1+\left(\frac{x}{\alpha}\right)^{\beta}\right]\right\}^{1-a}\left[1+\left(\frac{x}{\alpha}\right)^{\beta}\right]^{-1}-g(x)}=\frac{2 \beta}{\alpha^{\beta}} x^{\beta-1}\left[1+\left(\frac{x}{\alpha}\right)^{\beta}\right]^{-1}, x>0 .
$$

Remark 4.3. The general solution of the differential equation given in Corollary 4.1 is

$$
\eta(x)=\left[1+\left(\frac{x}{\alpha}\right)^{\beta}\right]^{2}\left[\begin{array}{c}
-\int g(x) \frac{\beta}{\alpha^{\beta}} x^{\beta-1}\left[1+\left(\frac{x}{\alpha}\right)^{\beta}\right]^{-2} \\
\left\{\log \left[1+\left(\frac{x}{\alpha}\right)^{\beta}\right]\right\}^{a-1} d x+D
\end{array}\right],
$$

for $x \in \mathbb{R}^{+}$, where $D$ is a constant. One set of appropriate functions is given in Proposition 4.1 with $D=0$.

\subsection{Based on truncated moment of certain functions of the $n^{\text {th }}$ order statistic}

Let $X_{1: n}, X_{2: n}, \ldots, X_{n: n}$ be $n$ order statistics from a continuous cdf $F$. We state here a characterization result based on certain functions of the $n^{\text {th }}$ order statistic. Our characterization of ZBLL here will be a consequence of the following proposition, which is similar to the one appeared in our previous work (Hamedani, [14]).

Proposition 4.2. Let $X: \Omega \rightarrow(0, \infty)$ be a continuous random variable with cdf $F$. Let $\psi(x)$ and $q(x)$ be two differentiable functions on $(0, \infty)$ such that $\lim _{x \rightarrow 0} \psi(x)[F(x)]^{n}=0$ and $\int_{0}^{\infty} \frac{q^{\prime}(t)}{[\psi(t)-q(t)]} d t=\infty$. Then, $E\left[\psi\left(X_{n: n}\right) \mid X_{n: n}<t\right]=q(t)$ for $t>0$ implies

$$
F(x)=\exp \left\{-\int_{x}^{\infty} \frac{q^{\prime}(t)}{n[\psi(t)-q(t)]} d t\right\}, \quad x \geq 0
$$

Remark 4.4. Taking, e.g., $\psi(x)=\left(\gamma\left[\alpha, \log \left(1+\left(\frac{x}{\alpha}\right)^{\beta}\right)\right]\right)^{n \alpha}$ and $q(x)=\frac{1}{2} \psi(x)$ in Proposition $4.2,(4.1)$ will reduce to the cdf $(2.3)$.

\subsection{Based on truncated moment of certain functions of the $1^{\text {st }}$ order statistic}

We state here a characterization result based on certain functions of the $1^{\text {st }}$ order statistic. Our characterization of ZBLL here, for $\alpha=1$, will be a consequence of the following proposition, which is similar to the one appeared in our previous work (Hamedani, [14]).

Proposition 4.3. Le $X: \Omega \rightarrow(0, \infty)$ be a continuous random variable with cdf $F$. Let $\psi_{1}(x)$ and $q_{1}(x)$ be two differentiable functions on $(0, \infty)$ such that $\lim _{x \rightarrow \infty} \psi_{1}(x)[1-F(x)]^{n}=0$ and 
$\int_{0}^{\infty} \frac{q_{1}^{\prime}(t)}{\left[q_{1}(t)-\psi_{1}(t)\right]} d t=\infty$. Then, $E\left[\psi_{1}\left(X_{1: n}\right) \mid X_{1: n}>t\right]=q_{1}(t)($ for $t>0)$ implies

$$
F(x)=1-\exp \left\{-\int_{0}^{x} \frac{q_{1}^{\prime}(t)}{n\left[\psi_{1}(t)-q_{1}(t)\right]} d t\right\}, \quad x \geq 0
$$

Remark 4.5. Taking, e.g., $\psi_{1}(x)=2\left(1+x^{\beta}\right)^{n}$ and $q_{1}(x)=\frac{1}{2} \psi_{1}(x)$ in Proposition 4.3, (4.2) will reduce to the $\operatorname{cdf}(2.3)$ with $\alpha=1$.

\section{Order statistics}

Order statistics make their appearance in many areas of statistical theory and practice. Suppose $X_{1}, X_{2}, \ldots, X_{n}$ is a random sample from the ZBLL distribution. Let $X_{i: n}$ denote the $i$ th order statistic. From equation (2.5) and the corresponding cdf, the pdf of $X_{i: n}$ becomes

$$
\begin{aligned}
f_{i: n}(x) & =K f(x) F^{i-1}(x)\{1-F(x)\}^{n-i}=K \sum_{j=0}^{n-i}(-1)^{j}\left(\begin{array}{c}
n-i \\
j
\end{array}\right) f(x) F^{j+i-1}(x) \\
& =K \sum_{j=0}^{n-i}(-1)^{j}\left(\begin{array}{c}
n-i \\
j
\end{array}\right)\left[\sum_{r=0}^{\infty} b_{r}(a+r) G(x)^{a+r-1} g(x)\right]\left[\sum_{k=0}^{\infty} b_{k} G(x)^{a+k}\right]^{j+i-1}
\end{aligned}
$$

where $K=n ! /[(i-1) !(n-i) !]$. Using the power series raised to a positive integer, we can write

$$
\left[\sum_{k=0}^{\infty} b_{k} G(x)^{a+k}\right]^{j+i-1}=\sum_{k=0}^{\infty} f_{j+i-1, k} G(x)^{a(j+i-1)+k},
$$

where $f_{j+i-1,0}=b_{0}^{j+i-1}$ and (for $k=1,2, \ldots$ )

$$
f_{j+i-1, k}=\left(k b_{0}\right)^{-1} \sum_{m=1}^{k}[m(j+i)-k] b_{m} f_{j+i-1, k-m} .
$$

Hence,

$$
f_{i: n}(x)=\sum_{j=0}^{n-i} \sum_{r, k=0}^{\infty} m_{j, r, k} h_{a(j+i)+r+k}(x),
$$

where

$$
m_{j, r, k}=\frac{(-1)^{j} n !}{(i-1) !(n-i-j) ! j !} \frac{(a+r) b_{r} f_{j+i-1, k}}{[a(j+i)+r+k]} .
$$

Equation (5.1) reveals that the density function of the ZBLL order statistics can be expressed as a triple linear combination of ELL densities. So, several mathematical properties of $X_{i: n}$ can be obtained from the corresponding ELL properties. 


\section{Maximum likelihood estimation}

Here, we consider the estimation of the unknown parameters of the ZBLL distribution by the method of maximum likelihood. Let $x_{1}, \ldots, x_{n}$ be a random sample of size $n$ from the $\operatorname{ZBLL}(a, \alpha, \beta)$ distribution. The log-likelihood function for the vector of parameters $\theta=(a, \alpha, \beta)^{T}$ can be expressed as

$$
\begin{aligned}
l(\theta) & =n \log (\beta)-n \beta \log (\alpha)-\log [\Gamma(a)]+(\beta-1) \sum_{i=1}^{n} \log \left(x_{i}\right)-2 \sum_{i=1}^{n}\left(1+\frac{x_{i}}{\alpha}\right) \\
& +(a-1) \sum_{i=1}^{n} \log \left\{\log \left[1+\left(\frac{x_{i}}{\alpha}\right)^{\beta}\right]\right\} .
\end{aligned}
$$

The components of the score vector $U(\theta)$ are given by

$$
\begin{gathered}
U_{a}(\theta)=-\psi(a)+\sum_{i=1}^{n} \log \left\{\log \left[1+\left(\frac{x_{i}}{\alpha}\right)^{\beta}\right]\right\} \\
U_{\alpha}(\theta)=-\frac{n \beta}{\alpha}+2 \sum_{i=1}^{n} \frac{x_{i}}{\alpha^{2}}+\frac{(-a+1) \beta}{\alpha} \sum_{i=1}^{n}\left(\frac{x_{i}}{\alpha}\right)^{\beta}\left[1+\left(\frac{x_{i}}{\alpha}\right)^{\beta}\right]^{-1}\left\{\log \left[1+\left(\frac{x_{i}}{\alpha}\right)^{\beta}\right]\right\}^{-1}, \\
U_{\beta}(\theta)=\frac{n}{\beta}-n \log (\alpha)+\sum_{i=1}^{n} \log \left(x_{i}\right)+(a-1) \sum_{i=1}^{n}\left(\frac{x_{i}}{\alpha}\right)^{\beta} \log \left(\frac{x_{i}}{\alpha}\right)\left[1+\left(\frac{x_{i}}{\alpha}\right)^{\beta}\right]^{-1} \\
\times\left\{\log \left[1+\left(\frac{x_{i}}{\alpha}\right)^{\beta}\right]\right\}^{-1},
\end{gathered}
$$

where $\psi(\cdot)$ is the digamma function.

The maximum likelihood estimate (MLE) $\widehat{\theta}$ of $\theta$ is obtained by solving the nonlinear likelihood equations $U_{a}(\theta)=0, U_{\alpha}(\theta)=0$ and $U_{\beta}(\theta)=0$. These equations cannot be solved analytically and statistical software can be used to solve them numerically. For interval estimation and hypothesis tests on the model parameters, we can calculate the $3 \times 3$ observed information matrix $J(\theta)=$ $-\left\{U_{r s}\right\}$, where $r, s=a, \alpha, \beta$, since its expectation requires numerical integration. The elements $U_{r s}$ are listed in Appendix C.

\section{Application}

We provide one application to real data to demonstrate the potentiality of the ZBLL distribution. The data represent the survival times of 121 patients with breast cancer obtained from a large hospital in a period from 1929 to 1938 (Lee, [17]). The data are:

$0.3,0.3,4.0,5.0,5.6,6.2,6.3,6.6,6.8,7.4,7.5,8.4,8.4,10.3,11.0,11.8,12.2,12.3,13.5,14.4,14.4$, 14.8, 15.5, 15.7, 16.2, 16.3, 16.5, 16.8, 17.2, 17.3, 17.5, 17.9, 19.8, 20.4, 20.9, 21.0,21.0, 21.1, 23.0, $23.4,23.6,24.0,24.0,27.9,28.2,29.1,30.0,31.0,31.0,32.0,35.0,35.0,37.0,37.0,37.0,38.0,38.0$, 
38.0,39.0,39.0,40.0,40.0,40.0,41.0,41.0,41.0,42.0,43.0,43.0,43.0,44.0,45.0,45.0, 46.0, 46.0, 47.0,48.0, 49.0,51.0, 51.0, 51.0, 52.0, 54.0,55.0,56.0,57.0,58.0,59.0,60.0,60.0,60.0,61.0,62.0, $65.0,65.0,67.0,67.0,68.0,69.0,78.0,80.0,83.0,88.0,89.0,90.0,93.0,96.0,103.0,105.0,109.0$, 109.0, 111.0, 115.0,117.0, 125.0, 126.0, 127.0,129.0,129.0,139.0,154.0.

We shall compare the ZBLL distribution with some other lifetime models with four, three and two parameters, namely:

- Kumaraswamy Log-Logistic (KLL) distribution (Cordeiro et al, [3]). Its pdf (for $x>0$ ) is given by

$$
f_{K L L}(x)=\frac{a b \gamma}{\alpha^{a \gamma}} x^{a \gamma-1}\left[1+\left(\frac{x}{\alpha}\right)^{\gamma}\right]^{-(a+1)}\left\{1-\left[1-\frac{1}{1+\left(\frac{x}{\alpha}\right)^{\gamma}}\right]^{a}\right\}^{b-1} ;
$$

- Beta Log-Logistic (BLL) distribution (Lemonte, [18]). Its pdf (for $x>0$ ) is given by

$$
f_{B L L}(x)=\frac{\beta}{\alpha \mathrm{B}(a, b)} \frac{\left(\frac{x}{\alpha}\right)^{a \beta-1}}{\left[1+\left(\frac{x}{\alpha}\right)^{\beta}\right]^{a+b}} ;
$$

- Exponentiated Weibull (EW) distribution (Mudholkar and Srivastava, [19]), which has pdf (for $x>0$ ) given by

$$
f_{E W}(x)=\frac{c \theta}{\alpha}\left(\frac{x}{\alpha}\right)^{c-1} \mathrm{e}^{-\left(\frac{x}{\alpha}\right)^{c}}\left[1-e^{-\left(\frac{x}{\alpha}\right)^{c}}\right]^{\theta-1}
$$

- Exponentiated Log-Logistic (ELL) distribution. Its pdf (for $x>0$ ) is given by

$$
f_{E L L}(x)=\frac{a \beta}{\alpha^{a \beta}} x^{a \beta-1}\left[1+\left(\frac{x}{\alpha}\right)^{\beta}\right]^{-(a+1)} .
$$

Table 1 lists the MLEs of the model parameters and the values of the following statistics: Akaike Information Criterion (AIC), Bayesian Information Criterion (BIC), Consistent Akaike Information Criterion (CAIC), Cramér-von Mises $\left(W^{*}\right)$ and Anderson-Darling $\left(A^{*}\right)$. The last two statistics are described by Chen and Balakrishnan [2]. They are used to verify which distribution fits better to the data. In general, the smaller the values of $W^{*}$ and $A^{*}$, the better the fit. Let $H(x ; \boldsymbol{\theta})$ be the cdf, where the form of $H$ is known but $\boldsymbol{\theta}$ (a $k$-dimensional parameter vector, say) is unknown. To obtain the statistics $W^{*}$ and $A^{*}$, we can proceed as follows: (i) Compute $v_{i}=H\left(x_{i} ; \widehat{\boldsymbol{\theta}}\right)$, where the $x_{i}$ 's are in ascending order; (ii) Compute $y_{i}=\Phi^{-1}\left(v_{i}\right)$, where $\Phi(\cdot)$ is the standard normal cdf and $\Phi^{-1}(\cdot)$ its inverse; (iii) Compute $u_{i}=\Phi\left\{\left(y_{i}-\bar{y}\right) / s_{y}\right\}$, where $\bar{y}=n^{-1} \sum_{i=1}^{n} y_{i}$ and $s_{y}^{2}=(n-1)^{-1} \sum_{i=1}^{n}\left(y_{i}-\bar{y}\right)^{2}$; (iv) Calculate $W^{2}=\sum_{i=1}^{n}\left\{u_{i}-(2 i-1) /(2 n)\right\}^{2}+1 /(12 n)$ and $A^{2}=-n-n^{-1} \sum_{i=1}^{n}\left\{(2 i-1) \log \left(u_{i}\right)+\right.$ $\left.(2 n+1-2 i) \log \left(1-u_{i}\right)\right\} ;(\mathrm{v})$ Modify $W^{2}$ into $W^{*}=W^{2}(1+0.5 / n)$ and $A^{2}$ into $A^{*}=A^{2}(1+$ $\left.0.75 / n+2.25 / n^{2}\right)$. 
The computations were performed using the package AdequacyModel in $\mathrm{R}$ developed by the authors Cícero Dias and Pedro Marinho. The figures of Table 1 indicate that the ZBLL model yields the smallest value of the statistics $W^{*}$ and $A^{*}$, and then it could be chosen as the best model among these distributions. More details can be provided by a visual comparison in Figure 4 of the histogram of the data and the fitted density functions of the ZBLL, BLL and EW distributions. The KLL model does not fit the data well and the curve of the ELL model almost coincides with the curve of the ZBLL model. Clearly, the ZBLL distribution provides a better fit to the histogram and hence it is the best model to explain these data.

Table 1. MLEs (standard errors in parentheses) and the statistics $W^{*}$ and $A^{*}$

\begin{tabular}{|c|c|c|c|c|c|c|c|c|c|}
\hline \multirow{2}{*}{$\begin{array}{l}\text { Distributions } \\
\operatorname{KLL}(a, b, \gamma, \alpha)\end{array}$} & \multicolumn{4}{|c|}{ Estimates } & \multirow{2}{*}{$\frac{\mathbf{A}^{*}}{1.511}$} & \multirow{2}{*}{$\frac{\mathbf{W}^{*}}{0.232}$} & \multirow{2}{*}{$\begin{array}{c}\text { AIC } \\
1189.937\end{array}$} & \multirow{2}{*}{$\begin{array}{c}\text { CAIC } \\
1190.282\end{array}$} & \multirow{2}{*}{$\begin{array}{c}\text { BIC } \\
1201.120\end{array}$} \\
\hline & 33.698 & 23.048 & 0.336 & 0.044 & & & & & \\
\hline & $(4.808)$ & (13.979) & $(0.043)$ & $(0.020)$ & & & & & \\
\hline \multirow[t]{2}{*}{$\operatorname{BLL}(a, b, \alpha, \beta)$} & 0.364 & 0.732 & 53.251 & 3.368 & 0.494 & 0.066 & 1171.861 & 1172.206 & 1183.045 \\
\hline & $(0.230)$ & $(0.482)$ & $(9.731)$ & (1.716) & & & & & \\
\hline \multirow[t]{2}{*}{$\operatorname{ZBLL}(a, \alpha, \beta)$} & 0.353 & 77.856 & 3.098 & & 0.454 & 0.053 & 1167.063 & 1167.268 & 1175.450 \\
\hline & $(0.103)$ & (12.562) & $(0.579)$ & & & & & & \\
\hline \multirow[t]{2}{*}{$\mathrm{EW}(c, \theta, \alpha)$} & 1.494 & 0.798 & 57.958 & & 0.456 & 0.061 & 1163.759 & 1163.964 & 1172.146 \\
\hline & $(0.395)$ & $(0.334)$ & (15.184) & & & & & & \\
\hline \multirow[t]{2}{*}{$\operatorname{ELL}(a, \alpha, \beta)$} & 0.321 & 70.715 & 3.401 & & 0.455 & 0.053 & 1167.341 & 1167.546 & 1175.728 \\
\hline & $(0.094)$ & $(10.458)$ & $(0.658)$ & & & & & & \\
\hline
\end{tabular}

\section{Concluding remarks}

In this paper, we propose a new distribution which generalizes the log-logistic distribution. The new model is called the Zografos-Balakrishnan log-logistic (ZBLL) distribution. The ZBLL distribution can have increasing, decreasing, bathlub and unimodal hazard rate functions. It is very versatile to fit lifetime data. We study some of its mathematical and statistical properties. We provide explicit expressions for the ordinary and incomplete moments, quantile and generating functions, mean deviations, Rényi entropy, reliability and some characterization results. The model parameters are estimated by maximum likelihood and the observed information matrix is determined. The potentiality of the new model is illustrated in an application using the script AdequacyModel in R. The ZBLL model can provide better fits than other common lifetime models. 


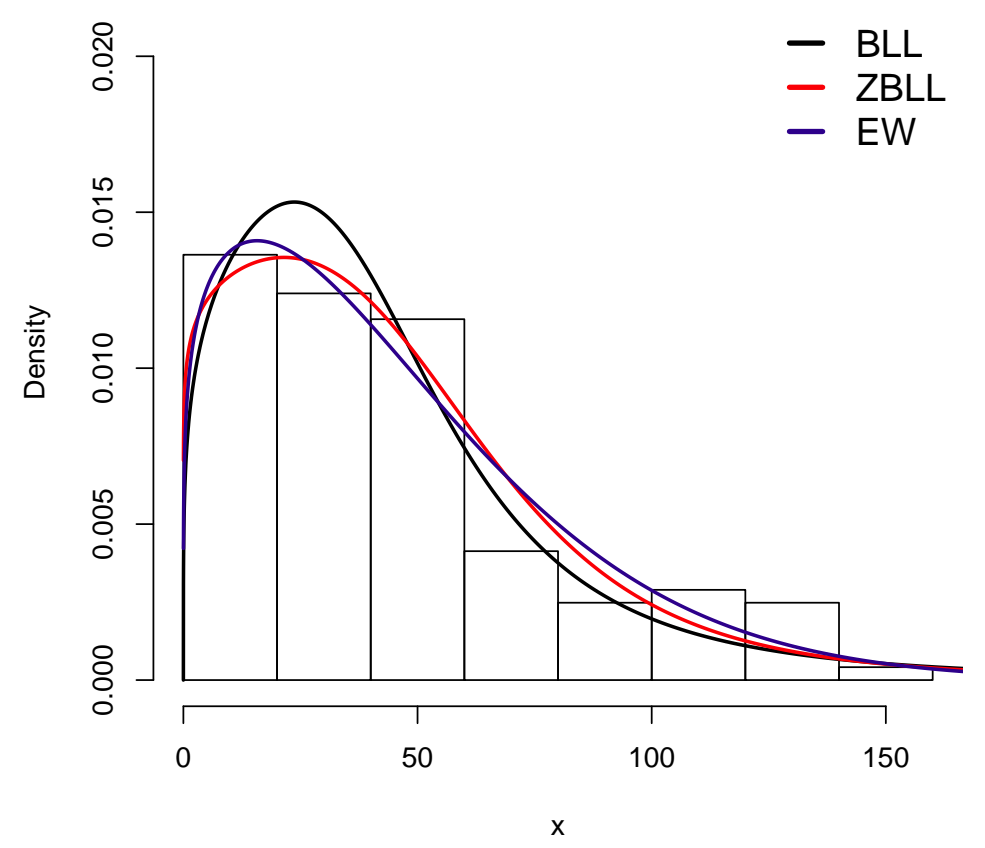

Fig. 4. Fitted Distributions.

\section{Appendix A - Rényi entropy}

The Rényi entropy of the ZBLL random variable (for $\gamma>0$ and $\gamma \neq 1$ ) is given by

$$
I_{R}(\gamma)=\frac{1}{(1-\gamma)} \log \int_{0}^{\infty} \frac{\beta^{\gamma} x^{(\beta-1) \gamma}}{\alpha^{\beta \gamma}[\Gamma(a)]^{\gamma}}\left[1+\left(\frac{x}{\alpha}\right)^{\beta}\right]^{-2 \gamma}\left\{\log \left[1+\left(\frac{x}{\alpha}\right)^{\beta}\right]\right\}^{(a-1) \gamma} d x .
$$

Using the expansion (for $\mathrm{a} \neq 1$ )

$$
\begin{aligned}
\left\{\log \left[1+\left(\frac{x}{\alpha}\right)^{\beta}\right]\right\}^{(a-1) \gamma} & =(a-1) \gamma \sum_{k=0}^{\infty}\left(\begin{array}{c}
k-\gamma a+\gamma \\
k
\end{array}\right) \sum_{j=0}^{k} \frac{(-1)^{j+k}\left(\begin{array}{l}
k \\
j
\end{array}\right) p_{j, k}}{[\gamma(a-1)-j]} \\
& \times\left\{1-\left[1+\left(\frac{x}{\alpha}\right)^{\beta}\right]^{-1}\right\}^{\gamma a-\gamma+k},
\end{aligned}
$$

we can rewrite $I_{R}(\gamma)$ as

$$
I_{R}(\gamma)=\frac{1}{(1-\gamma)} \log \left\{\frac{(a-1) \gamma}{[\Gamma(a)]^{\gamma}} \sum_{k=0}^{\infty}\left(\begin{array}{c}
k-\gamma a+\gamma \\
k
\end{array}\right) \sum_{j=0}^{k} \frac{(-1)^{j+k}\left(\begin{array}{l}
k \\
j
\end{array}\right) p_{j, k}}{[\gamma(a-1)-j]} M_{k}\right\}
$$

where $M_{k}$ comes after some algebra as

$$
M_{k}=\beta^{\gamma-1} \alpha^{\gamma(2 a \beta+2 \beta-\gamma)+1} B\left(\frac{\gamma(\beta+1)-1}{\beta}, \frac{\gamma(\beta a-1)+\beta k+1}{\beta}\right) .
$$


Hence, we can obtain (3.6).

\section{Appendix B - Reliability}

From equation (2.5) and the corresponding cdf, we can write

$$
R=\sum_{j, k=0}^{\infty} c_{j k} \int_{0}^{\infty} H_{a_{2}+j}(x) h_{a_{1}+k}(x) d x=\sum_{j, k=0}^{\infty} c_{j k} R_{j k}
$$

where $c_{j k}$ is defined in Section 3.5 and $R_{j k}=\operatorname{Pr}\left(Y_{j}<Y_{k}\right)$ is the reliability between the independent random variables $Y_{j} \sim \operatorname{ELL}\left(a_{2}+j\right)$ and $Y_{k} \sim \operatorname{ELL}\left(a_{1}+k\right)$. Hence, the reliability of the ZBLL distribution is a linear combination of the ELL reliabilities. We have

$$
H_{a_{2}+j}(x)=\left[\frac{\left(\frac{x}{\alpha}\right)^{\beta}}{1+\left(\frac{x}{\alpha}\right)^{\beta}}\right]^{a_{2}+j} \quad \text { and } \quad h_{a_{1}+k}(x)=\frac{\beta\left(a_{1}+k\right)\left(\frac{x}{\alpha}\right)^{\beta\left(a_{1}+k\right)-1}}{\alpha\left[1+\left(\frac{x}{\alpha}\right)^{\beta}\right]^{a_{1}+k+1}},
$$

and then we can obtain $R_{j k}$ after some algebra and write

$$
R=\sum_{j, k=0}^{\infty} \frac{c_{j k}\left(a_{1}+k\right) \alpha^{-\beta\left(a_{1}+a_{2}+j+k+1\right)}}{\left(a_{1}+a_{2}+j+k\right)} .
$$

\section{Appendix C - Observed information matrix}

For $p, m, q, r \in\{0,1,2\}$, we define the transformed observation

$$
T_{p, m, q, r}\left(x_{i}\right)=\left(\frac{x_{i}}{\alpha}\right)^{p \beta}\left[\log \left(\frac{x_{i}}{\alpha}\right)\right]^{m}\left[1+\left(\frac{x_{i}}{\alpha}\right)^{\beta}\right]^{-q}\left\{\log \left[1+\left(\frac{x_{i}}{\alpha}\right)^{\beta}\right]\right\}^{-r} .
$$

The elements of the observed information matrix for the parameters $(a, \alpha, \beta)$ are given by:

$$
\begin{gathered}
U_{a a}=-\psi^{\prime}(a), U_{a \alpha}=-\beta \alpha^{-1} \sum_{i=1}^{n} T_{1,0,1,1}\left(x_{i}\right), U_{a \beta}=\sum_{i=1}^{n} T_{1,1,1,1}\left(x_{i}\right), \\
U_{\alpha \alpha}=\frac{n \beta}{\alpha^{2}}-4 \sum_{i=1}^{n} \frac{x_{i}}{\alpha^{3}}+\beta \alpha^{-2}[(1-1) \beta+1] \sum_{i=1}^{n} T_{1,0,1,1}\left(x_{i}\right) \\
-\beta^{2} \alpha^{-2} \sum_{i=1}^{n}\left[T_{2,0,2,1}\left(x_{i}\right)+T_{2,0,2,3}\left(x_{i}\right)\right], \\
U_{\alpha \beta}=-\frac{n}{\alpha}-(a-1) \beta \alpha^{-1} \sum_{i=1}^{n} T_{1,1,1,1}\left(x_{i}\right)-\alpha^{-1} \sum_{i=1}^{n} T_{1,0,1,1}\left(x_{i}\right) \\
+\beta \alpha^{-1} \sum_{i=1}^{n}\left[T_{2,1,2,1}\left(x_{i}\right)+T_{2,1,2,2}\left(x_{i}\right)\right], \\
U_{\beta \beta}=-\frac{n}{\beta^{2}}+(a-1) \sum_{i=1}^{n} T_{1,2,1,1}\left(x_{i}\right)-\sum_{i=1}^{n}\left[T_{2,2,2,1}\left(x_{i}\right)+T_{2,2,2,2}\left(x_{i}\right)\right],
\end{gathered}
$$

where $\psi^{\prime}(\cdot)$ is the trigamma function. 


\section{References}

[1] Ashkar, F., Mahdi, S. (2006). Fitting the log-logistic distribution by generalized moments. Journal of Hydrology, 328, 694-703.

[2] Chen, G., Balakrishnan, N. (1995). A general purpose approximate goodness-of-fit test. Journal of Quality Technology, 27, 154-161.

[3] Cordeiro, G.M., Santana, T.V.F., Ortega, E.M.M and Silva, G.O. (2012). The Kumaraswamy-LogLogistic distribution. Journal of Statistical Theory and Applications, 11, 265-291.

[4] Galambos, J. and Kotz, S. (1978). Characterizations of probability distributions. A unified approach with an emphasis on exponential and related models. Lecture Notes in Mathematics, 675, Springer, Berlin.

[5] Glänzel, W. (1987). A characterization theorem based on truncated moments and its application to some distribution families. Mathematical Statistics and Probability Theory (Bad Tatzmannsdorf, 1986), Vol. B, Reidel, Dordrecht, 75-84.

[6] Glänzel, W. and Hamedani, G.G. (2001). Characterizations of univariate continuous distributions. Studia Scientiarum Mathematicarum Hungarica, 37, 83-118.

[7] Glänzel, W., Telcs, A. and Schubert, A. (1984). Characterization by truncated moments and its application to Pearson-type distributions. Zeitschrift für Wahrscheinlichkeitstheorie und Verwandte Gebiete, 66, 173-183.

[8] Glänzel, W. and Win, I. (1994). A characterization tool for discrete distributions under Window $(R)$. In R. Dutter and W. Grossmann (Eds.), COMPSTAT 94 (Vienna, 1994), Short Communications in Computational Statistics, 199-200.

[9] Gupta, R.C., Gupta, P.L. and Gupta R.D. (1998). Modeling failure time data by Lehman alternatives. Communications in Statistics-Theory and Methods, 27, 887-904.

[10] Gupta, R.D. and Kundu, D. (1999). Generalized exponential distributions. Australian and New Zealand Journal of Statistics, 41, 173-188.

[11] Gupta, R.D. and Kundu, D. (2001). Generalized exponential distribution: Different methods of estimation. J. Stat. Comput. Simul., 69, 315-337.

[12] Hamedani, G.G. (2002). Characterizations of univariate continuous distributions II. Studia Scientiarum Mathematicarum Hungarica, 39, 407-424.

[13] Hamedani, G.G. (2006). Characterizations of univariate continuous distributions III. Studia Scientiarum Mathematicarum Hungarica, 43, 361-385.

[14] Hamedani, G.G. (2010). Characterizations of continuous univariate distributions based on the truncated moments of functions of order statistics. Studia Scientiarum Mathematicarum Hungarica, 47, 462-484.

[15] Kleiber, C. and Kotz, S. (2003). Statistical Size Distributions in Economics and Actuarial Sciences. John Wiley: New York.

[16] Kotz, S. and Shanbhag, D.N. (1980). Some new approaches to probability distributions. Advances in Applied Probability, 12, 903-921.

[17] Lee, E.T. (1992) Statistical Methods for Survival Data Analysis. John Wiley: New York. 
M. W. A. Ramos et al.

[18] Lemonte, A.J. (2012). The beta log-logistic distribution. Brazilian Journal of Probability and Statistics. Accepted.

[19] Mudholkar, G.S. and Srivastava, D.K. (1993). Exponentiated Weibull family for analyzing bathtub failure-rate data. IEEE Transactions on Reliability, 42, 299-302.

[20] Mudholkar, G.S., Srivastava, D.K. and Freimer, M. (1995). The exponentiated Weibull family: A reanalysis of the bus-motor-failure data. Technometrics, 37, 436-445.

[21] Nadarajah, S. (2005). The exponentiated Gumbel distribution with climate application. Environmetrics, 17, 13-23.

[22] Nadarajah, S. and Kotz, S. (2006). The exponentiated type distributions. Acta Applicandae Mathematicae, 92, 97-111.

[23] Nadarajah, S., Cordeiro, G.M., Ortega, E.M. (2013). The gamma-G family of distributions: Mathematical properties and applications. Communications in Statistics Theory and Methods. To Appear.

[24] Ristic, M.M., Balakrishnan, N. (2012). The Gamma Exponentiated Exponential Distribution. Journal of Statistical Computation and Simulation, 82, 1191-1206.

[25] Tadikamalla, P.R. (1980). A look at the Burr and related distributions. International Statistical Review, 48, 337-344.

[26] Zografos, K. and Balakrishnan, N. (2009). On families of beta- and generalized gamma-generated distributions and associated inference. Statistical Methodology, 6, 344-362. 